This is a pre-copyedited version of a contribution published in Johansson D., Bagge H., Wahlström A.. (eds) Cold Climate HVAC 2018. CCC 2018. Springer Proceedings in Energy. Springer, Cham. https://doi.org/10.1007/978-3-030-00662-4_60

\title{
Fast simulation platform for retrofitting measures in residential heating
}

\author{
Philipp Schuetz ${ }^{*}, 1$ [0000-0001-7564-1468], Rossano Scoccia² ${ }^{[0000-0002-5894-7198] ~}$ \\ Damian Gwerder ${ }^{1}$, Remo Waser ${ }^{1,[0000-0002-4246-719 X]}$, David Sturzenegger ${ }^{1}$, Peru El- \\ guezabal $^{3}$, Beñat Arregi ${ }^{3,[}$ 0000-0002-9029-1678], Alessandro Sivieri',[0000-0001-8501-5401],

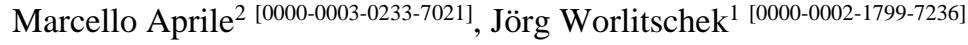 \\ ${ }^{1}$ Lucerne University of Applied Sciences and Arts, School of Engineering and Architecture, \\ Technikumstrasse 21, CH-6048 Horw \\ 2 Politecnico di Milano - Department of Energy, Via Lambruschini 4a, IT-20156 Milano \\ ${ }^{3}$ Tecnalia, Sustainable Construction Division, Parque Tecnológico de Bizkaia, ES-48160 Derio \\ schutz.p@gmail.com
}

\begin{abstract}
Energy efficiency aware building owners are facing a massive amount of different retrofitting options. However, a quantitative assessment of the different options requires a high level of technical expertise.

In this contribution, a fast and novel simulation platform for the assessment of different residential heating system configurations is presented. This platform enables dynamic simulations of the complete heating system, calculating energy/heat consumption and comfort indicators for different heating systems during a full year in less than 5 seconds on a recent laptop. Another key feature of the platform is the inclusion of a large variety of different heat sources (oil/gas/biomass/carbon boilers, air/brine-water or sorption heat pumps), sensible thermal heat storages, as well as building models. Shortly, this system will be the core of a platform enabling interested users to calculate the energy consumption of different retrofitting options accurately.

To validate the system models, the energy consumption of the three reference buildings (single family houses with an annual heating energy demand of 15, 45 and $100 \mathrm{kWh} / \mathrm{m}^{2}$ ) as per the IEA SHC Task 44 is calculated and compared with reference simulations from established simulation frameworks. The energy consumption of these buildings matches the reference values up to $5 \%$ for a full year simulation requiring calculations times between 3.3 and 3.7 seconds on a recent laptop.
\end{abstract}

Keywords: Assessment of retrofitting measures in residential heating, fast simulation platform, economic and ecological assessment tool.

\section{Introduction}

In 2013, around $22 \%$ of the overall energy consumption in EU28 was employed for space heating of residential buildings [1]. A reduction of this energy demand often requires a retrofitting of the considered buildings. Despite partially high incentives for renovation, the annual renovation rate in the European Union is currently rather low 
with around $3.6 \%$ estimated [2]. One burden to motivate building owners for retrofitting is the low dissemination of reliable information about different retrofitting options as well as a lack of a platform for comparing different alternatives. However, establishing such a platform poses two competing challenges. On the one hand, the comparison tool should be fast in estimating the energy consumption of the owner's specific building. On the other hand, the estimated consumption values must be reliable/accurate to enable a fair comparison of the different options, and the user must enter many parameters. To estimate the energy consumption over one year, simulations attracted much attention [3-5]. In order to enable simulations of the complete heating system over a long period, often detailed models of individual devices are simplified by analytical models $[3,6,7]$ and combined to models of the complete heating system. The major difference between the different approaches is the number of components considered, the complexity of the building and its model and the options of control (three position valve control [3] to complex model predictive control [8]) and the state of validation. The reference frameworks employed for benchmarking include TRNSYS, Matlab/Simulink (Carnot Blockset) [9] and IDA Indoor Climate and Energy framework[10].

In this contribution, a novel framework for the fast assessment of the energy consumption for residential heating systems is presented. Two major applications are envisioned: First, integrated in an online tool, interested users can assess the effect of different retrofitting measures for their situation (location, climate, energy pricing schemes, heating/cooling system). Second, expert users will employ this tool to investigate the effect of different system configurations, size components and quantify the effect of different control options. This contribution is organised as follows: In section 2 , the model platform and the models of the individual components are described. In section 3 , the validation results and performance evaluations of the simulation platform are presented. In the concluding section 4 , a conclusion of the obtained results is drawn.

\section{Methods}

\subsection{Modelling overview}

The dynamics of the residential heating system is described by combining numeri$\mathrm{cal} /$ analytical models of the individual components. For each component, a set of observable functions (e.g. the average temperatures in a pipe) is selected. Their evolution is either described by a differential equation derived from an energy balance equation (pipe, building, sensible thermal storage) or by a lookup table (heat pump) or an analytical function (oil/gas/biomass boiler, emitter system, valves).

\subsection{Models}

\section{Pipes}

As observable function the average temperature $T_{p}$ in the pipe is chosen. From the energy conservation equation, the following governing equation for the temperature evolution is derived: 


$$
m_{p} c_{m} \frac{\partial T_{p}}{\partial t}=\dot{Q}_{\text {in }}+\dot{m}_{\text {in }} c_{m} T_{\text {in }}-\dot{m}_{\text {out }} c_{m} T_{\text {out }}-\dot{Q}_{\text {loss }}
$$

with $m_{p}$ the mass of the medium in the pipe, $c_{m}$ the specific heat capacity, $T_{i n}, T_{\text {out }}$ and $\dot{m}_{\text {in }}, \dot{m}_{\text {out }}$ the temperature and mass flow rate of the medium at the inlet and outlet, respectively. With $\dot{Q}_{i n}$ the energy generated by heat sources connected to the pipe inlet is considered. The losses $\dot{Q}_{\text {loss }}$ are neglected as short, well insulated pipes are assumed.

\section{Building}

The complex dynamics of the building is modelled by a lumped energy balance equation of the building following the work of Burmeister et al. [11]. The change of the room temperature $T_{\text {room }}$ is described

$C \frac{\partial T_{\text {room }}}{\partial t}=-H\left(T_{\text {room }}-T_{\text {ambient }}\right)+g I+\dot{Q}_{E S}+\dot{Q}_{\text {internal }}$

by the action of internal losses (parametrised by the loss factor $\mathrm{H}$ and the difference between room temperature $T_{\text {room }}$ and the ambient temperature $T_{\text {ambient }}$ ), the contribution of solar radiation ( $g$ the solar factor considering window area and orientation and $I$ the instantaneous diffuse and direct radiation incident on the windows) as well as the heat transmitted via the emitter system $\dot{Q}_{E S}$ and internal gains $\dot{Q}_{\text {internal }}$ from electric/thermal appliances and inhabitants.

The contribution of the diffuse and direct radiation to the transmitted radiation $I$ is calculated based on the Perez sky model [12] and the implementation is adapted from the Carnot framework for Matlab [9]. The shading of the windows is modelled by adjusting the solar factor $\mathrm{g}$ and the effect of air exchange is modelled by additional loss terms in the loss factor $H$.

\section{Oil/Gas/Biomass boiler system}

The boiler system model has been implemented according to the European Standard CEN EN 15316-4-1 [13] following the "boiler cycling method" to incorporate the losses during cycling explicitly. This method calculates for each time step the instantaneous generated heat, the consumed energy carrier (oil, gas or biomass depending on the type) based on the current return temperature, and the operation state (burner on/off, loading factor and control strategy). The generated heat is then injected into the heating system analogous to the heat pump model presented above.

\section{Heat pump}

The heat pump is modelled based on a performance map approach. For each integration step, the current generated heat and coefficient of performance (COP) of the considered heat pumps is determined based on supply and ambient- / brine-temperature by linear interpolation for on/off-controlled heat pumps. For capacity-controlled heat pumps, in addition, the current load status is considered as a third dimension in the performance and COP maps. In the studies reported below, the performance map of an air/water- 
heat pump from Emerson Climate Technologies model ZHI-14 is considered. This heat pump has a thermal design power of $8091 \mathrm{~W}$ at the design air temperature of $-10{ }^{\circ} \mathrm{C}$ and a design outlet temperature of $55^{\circ} \mathrm{C}$. The performance and COP maps are shown in Fig. 1
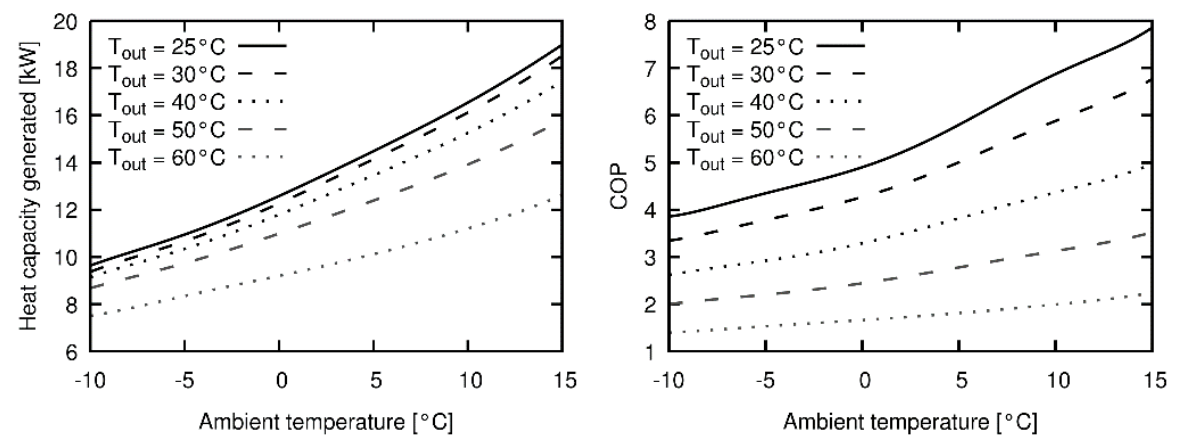

Fig. 1. Heating capacity generated (heating power) and coefficient of performance (COP) of considered air-water heat pump ZHI-14 from Emerson Climate technologies.

\section{Emitter system}

To model both radiator and underfloor-emitter systems, the heat $\dot{Q}_{\text {room }}$ exchanged with the room is calculated with the radiator equation:

$$
\dot{Q}_{\text {room }}=\dot{Q}_{\text {design }} \cdot\left(\frac{\frac{T_{\text {in }}+T_{E S}}{2}-T_{\text {room }}}{\Delta T_{\text {design }}}\right)^{n_{r}}
$$

with $\dot{Q}_{\text {design }}$ the design power of the emitter system at the design temperature difference $\Delta T_{\text {design }}$ between inlet temperature $T_{\text {in }}$, room temperature $T_{\text {room }}$ and the outlet temperature in the emitter system $T_{E S}$. The value radiator exponent $n_{r}$ enables to model a radiator based system $\left(n_{r}=1.3\right)$ and an underfloor based system $\left(n_{r}=1.1\right)$. To capture the inertia of the emitter system, the dynamics of the emitter system is modelled analogous to a pipe with extracted heat $\dot{Q}_{\text {room }}$ by the following equation:

$$
m_{E S} c \frac{\partial T_{E S}}{\partial t}=-\dot{Q}_{\text {room }}+\dot{m}_{\text {in }} c_{m} T_{\text {in }}-\dot{m}_{\text {in }} c_{m} T_{E S}
$$

In this equation, the mass flow $\dot{m}_{i n}$ into and out of the emitter system of the medium with capacity $c_{m}$ is assumed to be equal.

\section{Valves}

The effect of three-point valves is modelled by introducing the mass conservation as a constraint in the mass flow equations. To model a temperature control function, the splitting of the mass flows to maintain the target temperature is added as additional constraint equation. 


\section{Sensible thermal storage}

The sensible thermal storage is modelled as a vertical standing vessel with constant cross-section $A$ and height $h$. The height of the system is split into $N$ equal slices of height $\Delta h=h / N$. The mass flows from inlet and outlet pipe to the storage cause internal mass flows $\dot{m}_{u p}(i)$ (upwards) and $\dot{m}_{\text {down }}(i)$ (downwards) in the slices $i$ between inlet and outlet channels depending on the flow direction. For instance, an inlet mass flow of $1 \mathrm{l} / \mathrm{s}$ in a storage vessel with a height of $h=1 \mathrm{~m}$ discretized in $N=11$ slices, where the inlet enters at a height of $10 \mathrm{~cm}$ and exits at a height of $50 \mathrm{~cm}$, causes a mass flow upwards of $11 / \mathrm{s}$ for the nodes at 10, 20,30, 40 and $50 \mathrm{~cm}$. The dynamics within the storage vessel is captured via the evolution of the average temperature $T_{i}$ in the slice $i$ given by the following energy conservation equation:

$$
\begin{aligned}
m_{i} c_{m} \frac{\partial T_{i}}{\partial t}=U & \cdot A_{\text {loss }} \cdot\left(T_{\text {ambient,storage }}-T(i)\right) \\
& +\frac{\pi d^{2}}{4} \lambda_{F} \frac{T(i+1)+T(i-1)-2 T(i)}{\Delta h^{2}}+\dot{Q}_{\text {in }}(i) \\
& +c_{p}\left(\dot{m}_{u p}(i)(T(i-1)-T(i))+\dot{m}_{\text {down }}(i)(T(i+1)-T(i))\right)
\end{aligned}
$$

with $A_{\text {loss }}$ the surface of the slice in contact with the environment at temperature $T_{\text {ambient,storage }}, \lambda_{F}$ the heat conduction coefficient between adjacent slices, $\dot{Q}_{i n}(i)$ the heat injected in slice $i$ by external sources such as heat exchanges and $\dot{m}_{u p / \text { down }}(i)$ the mass flows in slice $i$ in upward and downward direction, respectively. For the nodes at the bottom and at the top of the storage vessel also the losses through the lids are incorporated with a term analogous to the term on the first line on the right. Effects of free convection within the storage vessel are not directly modelled but may be taken into consideration by the adaption of the thermal conductivity between adjacent slices.

\subsection{Calculation of key performance indicators}

\section{Energy consumption}

The energy consumption of the residential heating system is calculated by integrating the power injected by the heat source into the heating system. As described in detail in Section 2.4, the integration is performed stepwise, i.e., every 180 seconds, the instantaneous power of the heat source is multiplied with the width of the integration time window (unless the remaining simulation time is shorter, an integration interval of 180 seconds is chosen) and summed over the whole simulation period.

\section{Consumption of energy carrier}

The consumption of the energy carrier for the heat source is determined analogously to the energy consumption by stepwise integration of the instantaneous consumption. For the model of the oil/gas/biomass boiler, the instantaneous consumption of the energy carrier is calculated by the model according to EN norm 15316-4-1. In the heat pump model, the instantaneous, average coefficient of performance is determined by an interpolation of the provided COP map. By a division of the heat generated instantaneously and the current COP, the current electrical power consumption can be determined. 


\section{Comfort indicators}

As comfort indicator, the integrated deviation of the room temperature from the set temperature is considered. To incorporate the human insensitivity to deviations of $0.5 \mathrm{~K}$, the difference is only considered if the room temperature is $0.5 \mathrm{~K}$ below the actual set temperature.

\subsection{Simulation framework}

The simulation framework is implemented in C++ with Visual Studio Community 2017 from Microsoft. The models of the individual components are implemented in individual classes.

The simulation is performed in three stages:

1. The hydraulic configuration of the system is defined, all component models are loaded with the component parameters and required input and output values of the individual models are connected among the different component models by pointer algebra.

2. The dynamic simulation is performed: The whole simulation time (typically a full year) is split into small windows of 180 seconds. During this window the ambient temperature, the mass flow through the individual components and the control signals for the components are assumed to be constant. The dynamics of the heating system during this period is simulated by solving the system of coupled differential equations with an implementation of the controlled Runge-Kutta algorithm from the odeint package from the Boost 1.6.4 library (www.boost.org).

After each 180 second-window, the mass flows through the individual components are recalculated to account for changes in the operation conditions (e.g. changes in the mixing ratio of a temperature controlled mixing valve). The mass flows are determined by solving the system of mass conservation equations in each point of the hydraulic schema with the Eigen library (eigen.tuxfamily.org). In addition to the mass flow update, the climatic information is updated, changes in the control signal for the individual components are determined, and the changes on the key performance indicators (cf. Sections 2.3) are calculated.

3. In the last stage, the key performance indicators for the simulation are calculated, and the simulation program is terminated.

\subsection{Reference simulations for the building models}

For the benchmark of the building model, the reference buildings single family house SFH15, 45 and 100 from the IEA SHC Task 44 are considered [14, 15]. These three reference buildings are models for typical buildings with an annual space heating demand of 15,45 and $100 \mathrm{kWh} / \mathrm{m}^{2}$ and represent a modern energy efficient building (SFH15), a conventional, modern house (SFH45) and an existing, non-renovated building (SFH100) located in Strasbourg. To determine the annual energy demand for the models, a simple residential heating system is implemented in the framework: A heat source with constant power output (if running) is connected to the emitter system, 
which provides the energy to the building for space heating via the term $\dot{Q}_{E S}$. The usage profiles and contributions of the appliances $\dot{Q}_{\text {internal }}$ are taken from the reference publication $[14,15]$.

The simulations are performed for the climatic test reference year (TRY) of Strasbourg as extracted from Meteonorm. To measure the energy demand of the building alone, the heat source is exchanged with a constant power heat source injecting the same power into the heating system whenever activated. The shading control and air exchange are implemented as described in $[14,15]$.

In the building validation simulation, the heat source is controlled by a two position controller based on the current room temperature (set temperature $20^{\circ} \mathrm{C}$ with a death band of $1 \mathrm{~K})$.

To obtain time resolved reference energy consumption profiles of the building during one year, the energy consumption is as well simulated with the two reference software packages Matlab/Simulink (Carnot Blockset) [9] and IDA Indoor Climate and Energy framework (EQUA Simulation AB, Solna, Sweden). These reference simulations have been described elsewhere in detail [16].

\section{$3 \quad$ Results}

\subsection{Parametrisation of reference buildings and validation results}

Based on the detailed description of the buildings in $[14,15]$, the values of the lumped losses $H$, the summative building capacity $C$ and the solar gain factor $g$ have been calculated according to the procedure in [11]. The resulting values are reproduced in Table 1 together with the parameters of the emitter system and heat source in the simple benchmarking heating system.

\begin{tabular}{llll}
\hline Parameter & SFH 15 & Building & \\
\hline Loss factor $H(\mathrm{~W} / \mathrm{K})$ & 94.2 & 160.7 & SFH 100 \\
Capacity $C(\mathrm{~kJ} / \mathrm{K})$ & 169640 & 167123 & 163349 \\
Solar gain factor $g\left(\mathrm{~m}^{2}\right)$ & 11.5 & 12.2 & 14.8 \\
\hline Radiator exponent $n_{r}(-)$ & 1.1 & 1.1 & 1.3 \\
Design power $\dot{Q}_{\text {design }}(\mathrm{W})($ Strasbourg) & 1792 & 4072 & 7337 \\
Design temperature difference $\Delta T_{\text {design }}(\mathrm{K})$ & 15 & 15 & 35 \\
\hline Maximum power of heating system $(\mathrm{W})$ & 2330 & 5293 & 9538 \\
\hline Ventilation $\left(\mathrm{h}^{-1}\right)$ & 0.4 & 0.4 & 0.4 \\
\hline Heat exchanger effectiveness & 0.6 & 0 & 0 \\
\hline
\end{tabular}

Table 1. Parameters of building models according to Eq. (1), emitter system and heat source for the validation of the reference buildings SFH 15, SFH 45 and SFH 100 considered here.

The calculated cumulative weekly heat demand of the building for one year is shown in Fig. 2 and compared to the reference models. Comparing the three profiles indicates that all three simulations agree. The deviations of the annual space heat demand range between $1.2 \%$ (SFH15), $6.2 \%$ (SFH45) and $8.5 \%$ (SFH100). Although this deviation 
is non-negligible, it is compatible with the variation among the reference codes (e.g. $11.7 \%$ for SFH100). Noteworthily, all simulations of the current model have been performed with a focus on precise temperature control in the building: The comfort indicator (the integrated deviation of the room temperature - when larger than $0.5 \mathrm{~K}$ ) ranged only between 5 and $12 \mathrm{Kh}$ over a full year $(8760 \mathrm{~h})$.
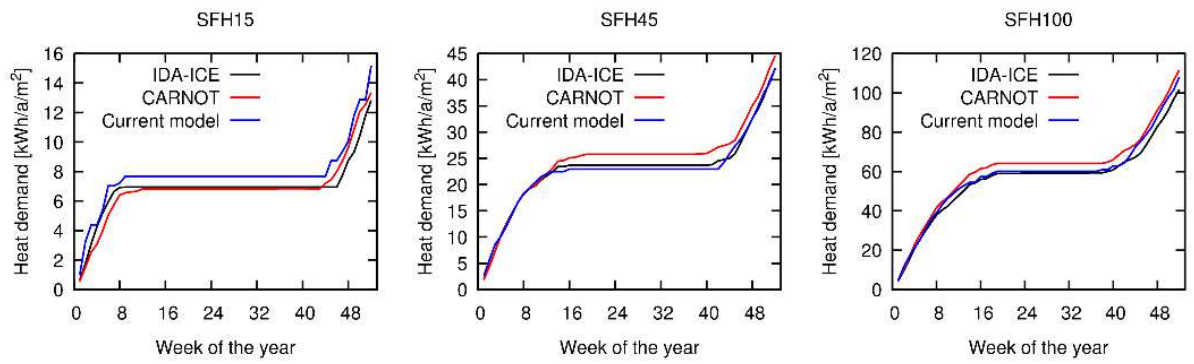

Fig. 2. Comparison of space heating energy demand for the three-considered reference building SFH15 (top, left), SFH45 (top, right) and SFH100 (bottom, left) for all considered simulation frameworks.

\begin{tabular}{llll}
\hline & \multicolumn{3}{c}{ Building } \\
Result & SFH 15 & SFH 45 & SFH 100 \\
\hline Annual energy demand $(\mathrm{kWh})$ & 2125 & 5909 & 15194 \\
Specific annual energy demand $\left(\mathrm{kWh} / \mathrm{m}^{2}\right)$ & 15.18 & 42.2 & 108.5 \\
Comfort indicator $(\mathrm{Kh})$ & 12 & 5 & 8.7 \\
Execution time for annual simulation $(\mathrm{s})$ & 3.54 & 3.66 & 3.61 \\
\hline
\end{tabular}

Table 2. Results of benchmarking study to evaluate annual space heating energy demand. The calculated specific annual energy demands match the expected values of 15,45 and $100 \mathrm{kWh} / \mathrm{m}^{2}$ accurately.

\subsection{Running time considerations}

The running times for the annual simulations are reported in Table 2 and range between 3.5 and 3.6 seconds. Due to the architecture tailored on speed, these running times are substantially lower than for the reference simulations, where annual simulations require execution times longer than 10 minutes.

\subsection{Application on residential heating system with different heating systems}

As a final application study, the building SFH100 is located in Helsinki (Meteonorm data set FI-Helsinki-Kaisani-29980). To cover the increased heat demand due to lower ambient temperatures, the power of the heat source has been adjusted appropriately (cf. Table 3 for reference). The (specific) energy consumption for the individual heat sources and the comfort indicators are shown in Table 3 as well. The constant power boiler and the condensing boiler are operated with a two level control based on the room temperature (set temperature $20^{\circ} \mathrm{C}$ ) and a dead band of $1 \mathrm{~K}$. The heat pump operation 
is controlled based on a set temperature on return temperature to the heat pump according to the heating curve modified by the six-fold deviation of room and set temperature.

\begin{tabular}{|c|c|c|c|}
\hline Property & $\begin{array}{l}\text { Fixed capacity } \\
\text { boiler }\end{array}$ & $\begin{array}{l}\text { Heat } \\
\text { pump }\end{array}$ & $\begin{array}{l}\text { Condensing } \\
\text { boiler }\end{array}$ \\
\hline $\begin{array}{l}\text { Power heat source at }-20{ }^{\circ} \mathrm{C} \text { at de- } \\
\text { sign point }(\mathrm{W})\end{array}$ & 10931 & 10911 & 10886 \\
\hline Heat generated per year $(\mathrm{kWh})$ & 29306 & 29387 & 29147 \\
\hline $\begin{array}{l}\text { Specific annual energy consump- } \\
\text { tion }\left(\mathrm{kWh} / \mathrm{m}^{2}\right)\end{array}$ & 209.3 & 209.9 & 208.19 \\
\hline $\begin{array}{l}\text { Annual final energy consumption } \\
(\mathrm{kWh})\end{array}$ & 29305 & 13284 & 31957 \\
\hline Comfort indicator $(\mathrm{Kh})$ & 368.8 & 46.3 & 436 \\
\hline $\begin{array}{l}\text { Execution time for annual simula- } \\
\text { tion }(s)\end{array}$ & 4.00 & 4.27 & 4.10 \\
\hline
\end{tabular}

Table 3. Annual specific energy demand and comfort indicators for an SFH 100 building in Helsinki.

Non-surprisingly, the heat demand for space heating almost doubled due to the change of location, which agrees with other simulation studies. The different control algorithms have only a minor impact on the total energy consumption. However, the heat pump control algorithm keeps the temperature in a narrower range yielding an improved value for the comfort indicator. For the considered heat pump, no performance data for ambient temperatures below $-10{ }^{\circ} \mathrm{C}$ are available. Therefore, the heat capacity and COP values for ambient temperatures below $-10^{\circ} \mathrm{C}$ have been derived from a linear extrapolation of the data in the range $0^{\circ} \mathrm{C}$ and $-10^{\circ} \mathrm{C}$. This may also be a reason for the rather high values of the seasonal performance factor of 2.19 calculated with the values above.

\section{Conclusions}

In this paper, a novel simulation platform for residential heating systems has been presented. The key features of this platform are its broad range of covered system components (different types of buildings, oil/gas/biomass boilers, heat pumps, different types of emitter systems as thermal storages) as well as its short execution time. These short execution times are vital for a fast assessment of different system configurations. To validate the building model, the simulation is compared with reference simulations performed with Matlab/Simulink (Carnot Blockset) and the IDA-Indoor Climate and Energy frameworks. The results of the framework agree well with the reference simulations for the SFH 15, 45 and 100 models as introduced by the IEA SHC Task 44. As an application, the energy consumption of the above three buildings has been compared for the different heat sources and different control algorithms with Helsinki and Strasbourg (reference location) as a point of installation. 


\section{Acknowledgements}

The authors would like to thank the European commission for funding of the H2020project "Heat4Cool" (project ID 723925). The work has also been supported by the Swiss State Secretariat for Education, Research and Innovation (SERI) under Contract No. 16.0082.

\section{References}

1. Pollier K, Gynther L, Lapillonne B (2015) Energy Efficiency Trends and Policies in the Household and Tertiary Sectors.

2. von Manteuffel, B. Petersdorff, C. Bettgenhäuser K, Boermans T (2016) EU pathways to a decarbonised building sector.

3. Byrne P, Miriel J, Lénat Y (2012) Modelling and simulation of a heat pump for simultaneous heating and cooling. Building Simulation 5:219-232.

4. Elci M, Narmsara S, Kagerer F, Herkel S (2013) Simulation of Energy Conservation Measures and Its Implications on a Combined Heat and Power District Heating System : a Case Study. In: 13th Conference of Building Performance Simulation Association. Chambéry, pp 104-111

5. Georges E, Masy G, Verhelst C, et al (2015) Smart Grid Energy Flexible Buildings Through The Use Of Heat Pumps In The Belgian Context. Science and Technology for the Built Environment 21:800-811.

6. Chung W (2010) Review of building energy-use performance benchmarking methodologies. Applied Energy 88:1470-1479.

7. Muratori M, Roberts MC, Sioshansi R, et al (2013) A highly resolved modeling technique to simulate residential power demand. Applied Energy 107:465-473.

8. Oldewurtel F, Parisio A, Jones CN, et al (2012) Use of model predictive control and weather forecasts for energy efficient building climate control. Energy and Buildings 45:15-27.

9. Wemhöner C, Hafner B, Schwarzer K (2000) Simulation of Solar Thermal Systems With Carnot Blockset. In: Proceedings Eurosun 2000 Conference, ISES, Copenhagen (DK), 1-6

10. EQUA IDA Indoor Climate and Energy.

11. Burmeister H, Keller B (1998) Climate surfaces: a quantitative building-specific representation of climates. Energy and Buildings 28:167-177.

12. Perez R, Ineichen P, Seals R, et al (1990) Modeling Daylight availability and irradiance components from direct and global irradiance. 44:271-289.

13. 15316-4-1 CE Heating systems in buildings: Method for calculation of system energy requirements and system efficiency: Part 4-1: Space heating generation systems, combustion systems (boilers).

14. Dott R, Ruschenburg J, Ochs F, et al (2013) The Reference Framework for System Simulation of the IEA SHC Task 44 / HPP Annex 38 - Part B: Buildings and Space Heat Load. Technical report of subtask $\mathrm{C}$ of the IEA SHC Task 44

15. Haller M, Ruschenburg J, Ochs F, et al (2013) The Reference Framework of System Simulations of the IEA SHC Task 44/HPP Annex 38 - Part A: General Simulation Boundary Conditions. Technical report of subtask $\mathrm{C}$ of the IEA SHC Task 44

16. Gwerder D, Schuetz P, Gasser L, et al (2015) Entwicklung einer optimalen Einheit aus Wärmepumpe und thermischem Energiespeicher. 21. Wärmepumpentagung BFE Forschungsprogramm 